\title{
Small molecule activation of the neurotrophin hepatocyte growth factor to treat Alzheimer disease
}

\author{
John W. Wright ${ }^{1,2}$, Joseph W. Harding ${ }^{1,2}$ \\ 'Department of Psychology, Washington State University, Pullman, WA 99164-4820, USA. \\ ${ }^{2}$ Department of Integrative Physiology and Neuroscience, and Program in Biotechnology, Washington State University, Pullman, \\ WA 99164-4820, USA.
}

Correspondence to: John W. Wright, Department of Psychology, Washington State University, P.O. Box 644820, Pullman, WA 99164-4820, USA. E-mail: wrightjw@wsu.edu How to cite this article: Wright JW, Harding JW. Small molecule activation of the neurotrophin hepatocyte growth factor to treat
Alzheimer disease. Neuroimmuno/ Neuroinflammation 2021;8:70-80. http://dx.doi.org/10.20517/2347-8659.2020.32

Received: 17 Apr 2020 First Decision: 8 Jul 2020 Revised: 14 Jul 2020 Accepted: 21 Jul 2020 Available online: 21 Jun 2021

Academic Editor: Daniele Orsucci Copy Editor: Cai-Hong Wang Production Editor: Jing Yu

\begin{abstract}
Alzheimer's disease (AD) is a neurodegenerative disease characterized by progressive neuron loses in memoryrelated brain structures. Five drugs have been approved by the FDA to treat Alzheimer's disease; however, these drugs have failed to modify or significantly slow disease progression. New therapies are needed to delay the course of this disease and hopefully prevent further neuron losses. This review describes available AD drugs and several novel approaches presently being investigated. We next describe relevant biomarkers and urge greater research interest in the potential utilization of neurotrophic agents to treat AD. Neurotrophins such as nerve growth factor, brain-derived neurotrophic factor and hepatocyte growth factor (HGF) are capable of stimulating dendritic arborization, synaptogenesis, stem cell differentiation, neurogenesis, and decreases in neuroinflammation, oxidative stress-induced damage and neurotoxicity due to a wide range of cellular insults. We present the strategy of utilizing small molecule analogs specifically designed to penetrate the blood-brain barrier and facilitate dimerization and activation of the HGF/Met receptor system. These molecules have been shown to encourage the formation of new functional synaptic connections, induce long-term potentiation and augment memory consolidation and retrieval in animal models of AD. Such molecules may be appropriate for use at the first indication of mild cognitive impairment, and perhaps prophylactically in those individuals who are most likely to develop dementia due to genetic, health, behavioral and life-style predisposing factors.
\end{abstract}

Keywords: Alzheimer's disease, brain-derived neurotrophic factor, hepatocyte growth factor, Trk receptor, Met receptor, angiotensin IV, dihexa

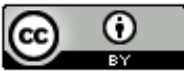

(C) The Author(s) 2021. Open Access This article is licensed under a Creative Commons Attribution 4.0 International License (https://creativecommons.org/licenses/by/4.0/), which permits unrestricted use, sharing, adaptation, distribution and reproduction in any medium or format, for any purpose, even commercially, as long as you give appropriate credit to the original author(s) and the source, provide a link to the Creative Commons license, and indicate if changes were made.

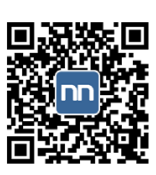




\section{INTRODUCTION}

Alzheimer's disease (AD) is a unique neurodegenerative disease causing progressive neuron damage in memory-associated structures, specifically neocortex, piriform cortex, hippocampus, locus ceruleus and the nucleus Basalis of Meynert. These areas display neuronal increases in amyloid- $\beta$ (A $\beta$ ) peptides and plaque formation, tau pathology, neuroinflammation, oxidative stress and gliosis ${ }^{[1-3]}$. AD patients also display additional biomarkers including cerebrovascular pathology and vascular dysfunctions, decreases in cerebral blood flow, cerebrovascular reactivity, and neurovascular coupling, leading to brain deficits in oxygen and nutrient delivery ${ }^{[4,5]}$.

In 1907 Alois Alzheimer described the symptoms presented by a 51-year old woman, Auguste Deter. These symptoms included progressive memory problems coupled with psychiatric disturbances. This patient died 5 years after diagnosis. Dr. Alzheimer indicated that her brain was atrophic with neuron losses and evidenced senile plaques and neurofibrillary tangles ${ }^{[6]}$. He went on to conclude that even though the presence of senile plaques and neurofibrillary tangles were observed, "the plaques are not the cause of senile dementia, but only an accompanying feature of senile involution of the central nervous system" ${ }^{[7]}$. Despite this warning research regarding AD has long been focused on the "amyloid cascade hypothesis" and strategies to prevent and/or remove plaques ${ }^{[2,8]}$. Unfortunately, the results seen from anti-amyloid (plaque buster) clinical trials have been disappointing ${ }^{[9]}$.

There are approximately 5.8 million $\mathrm{AD}$ patients in the U.S. and this number is predicted to reach 14-16 million by $2050^{[10]}$. The number of AD patients worldwide is expected to triple from 47 to 132 million by $2050^{[11]}$. Chronologically at age 60 the frequency of $\mathrm{AD}$ is approximately $1 \%$, at 65 years $10 \%$, at 85 years $30 \%$ and is now the sixth leading cause of death in the U.S. ${ }^{[10]}$. Annual U.S. treatment and care costs are presently $\$ 70-100$ billion $^{[12]}$ and worldwide more than $\$ 600$ billion $^{[13]}$, a number predicted to reach $\$ 3$ trillion by $2050^{[11]}$. A breakthrough in treatment is desperately needed otherwise the increasing patient numbers and associated treatment and care costs will severely impact our healthcare systems. Beyond these costs are the hardships and sacrifices suffered by family members and other caregivers, accompanied by the frustration experienced by health care professionals as patient cognitive abilities slowly decline.

This review describes the available FDA approved AD drugs, examples of drugs that have recently failed in clinical trials, and tentative reasons for these failures. There are several potential AD biomarkers thus far identified, and these are listed with several highlighted as particularly important. We recommend that at the present time electroencephalogram (EEG)-based measurements, including basal state analyses and event related potentials (ERPs), be used in clinical trials to evaluate new pro-cognitive therapeutics. Next, the many challenges associated with drug design are discussed and we urge additional research attention to neurotrophins given their demonstrated ability to address several of the pathologies that characterize AD. However, results have been poor because neurotrophins do not readily cross the blood-brain barrier and degrade rapidly in biological medium ${ }^{[14]}$. Over recent years two trials with nerve growth factor (NGF) designed to protect forebrain cholinergic neurons (early to moderate stage AD patients) were terminated after phase I ${ }^{[15,16]}$ (NCT00017940, NCT00876863). Other investigators have attempted to facilitate the availability of central brain-derived neurotrophic factor (BDNF) with injections of human BDNF directly into the patient's brain with minimal positive results ${ }^{[17]}$. Such discouraging findings have prompted research efforts directed at the development of small molecules configured to penetrate the blood-brain-barrier (BBB) and stimulate brain neurotrophins. Longo and colleagues have synthesized a small molecule BDNF mimetic that activates the TrkB receptor and facilitates motor learning in traumatic brain injured rats ${ }^{[18]}$. Members of our laboratory have developed small molecule hepatocyte growth factor (HGF) mimetics as discussed in the final section. These analogs have been shown to facilitate the formation of new functional synaptic connections, induce long-term potentiation (LTP), and augment memory consolidation and 
retrieval in animal models of AD. Thus, worldwide drug development efforts need re-evaluation and new strategies given that current efforts have been unsuccessful.

\section{FDA APPROVED DRUGS}

To date only two major drug categories have received FDA approval to treat Alzheimer disease despite intensive research efforts in the U.S. and around the world. Cholinesterase inhibitors including Razadyne, Exelon and Aricept, disrupt the degradation of acetylcholine thus extending the half-life and availability of this neurotransmitter acting at central cholinergic muscarinic and nicotinic receptors ${ }^{[19-21]}$. Namenda (memantine $\mathrm{HCl}$ ) acts as an $\mathrm{N}$-methyl-D-aspartate (NMDA) receptor antagonist designed to limit glutamate excitotoxicity and resulting neuronal damage ${ }^{[1,22,23]}$. A fifth drug, Namzaric, is a combination of Namenda and Aricept ${ }^{[10]}$.

None of these drugs delay the symptoms of AD nor do they prevent the progressive neurodegeneration seen in patients with this disease. There are several new drugs being evaluated designed to reduce cellular accumulations of $A \beta$ by inhibiting beta-secretase ${ }^{[24]}$, and employing monoclonal antibodies to $A \beta^{[25,26]}$. Although Roche and Eli Lilly have experienced drug failures in this regard, Biogen's antibody to A $\beta$, aducanumab (BIIB-037), has moved into Phase III clinical trials with promising results at low doses ${ }^{[27]}$. It must be noted that continuation of aducanumab to Phase III occurred only after a secondary data analyses suggesting some positive results. Tau aggregation inhibitors are also being tested to discourage neurofibrillary tangles ${ }^{[28]}$. Over the past 15 years more than 400 clinical trials of drugs to treat AD have been conducted ${ }^{[29]}$. Of those reporting outcomes nearly, all have shown little if any benefits ${ }^{[30]}$.

An efficacious treatment for $\mathrm{AD}$ has not been forthcoming in part due to the large numbers of potentially important biomarkers, the difficulty in determining their diagnostic reliability and validity, and the multifactorial characteristics of the disease process. Also, the established diagnostic indicators present in $\mathrm{AD}$ are seen in other forms of dementia including frontotemporal, vascular, diffuse Lewy body, corticobasal, dementia due to Parkinson's disease, type 2 diabetes, and HIV infection, as well as normal $\operatorname{aging}^{[31]}$. Several prominent investigators have speculated that the pathology of this disease initiates many years prior to the emergence of clinical symptoms ${ }^{[32-34]}$. Thus, research interest has focused on the development of early detection techniques via monitoring saliva, serum, CSF, neuroimaging biomarkers, and cognitive dysfunction ${ }^{[31]}$. Reliable detection at the earliest signs of AD related pathology could permit drug treatment many months or even years ahead of symptoms. Also, there is growing concern regarding concussion-induced cortical damage seen in children and young adults who participate in American football (chronic traumatic encephalopathy), boxing, martial arts, and soccer (due to headers) ${ }^{[35,36]}$. Evidence indicates that in severe cases repeated concussions can encourage mild cognitive impairment $(\mathrm{MCI})$, a predisposing condition often preceding $\mathrm{AD}^{[37]}$. The next section concerns the importance of biomarkers in the process of developing treatment strategies.

\section{BIOMARKERS FOR ALZHEIMER'S DISEASE}

Given the failure of amyloid plaque clearance drugs, and the likelihood that the current focus on tau aggregation inhibitors will also yield disappointing results, new therapeutic approaches are clearly needed to alter clinical outcome for Alzheimer's patients. These failures may be traced to the use of inappropriate biomarkers as guides to treatment development. Various biomarkers are not all equivalent and have differential value for disease diagnosis and drug development. The following represents a current definition of biomarkers. A biomarker is defined as a "characteristic that is measured as an indicator of normal biological processes, pathogenic processes, or responses to an exposure or intervention, including therapeutic interventions. It is not an assessment of how an individual feels, functions, or survives" ${ }^{\text {"[38] }}$. 
There are many potentially useful biomarkers presently proposed making them difficult to prioritize; however, there is reasonable agreement that with aging comes the increased likelihood of compromised innate immunity, oxidative stress-induced damage, infection and neuroinflammation accompanied by microgliosis ${ }^{[2,3,39-42]}$. With time microglia alter phenotype thus encouraging inflammation and neurodegeneration ${ }^{[40,43-45]}$. In addition, brain microglia-derived cytokines (IL-1 $\beta$, IL-6, IL-12, and TNF) become elevated and appear to facilitate disease progression ${ }^{[46]}$. Recently, neurofilament light chain has been proposed as a useful marker for $\mathrm{AD}$ as evidenced in Down syndrome patients prone to Alzheimer's disease ${ }^{[47]}$.

Some biomarkers, for example the accumulation of phospho-tau proteins, or the decrease in A $\beta 42$ levels in the CSF, serve as excellent diagnostic markers but may be less valuable as evaluation tools for therapeutic development. These alterations in levels have likely taken years to manifest resulting in well-established functional deficits. Thus, changes in these biomarkers do not accurately assess real-time brain function since the underlying and established deficits in neuronal functioning are unaffected by their absence. As such, they are not useful assessment tools for drug development. By contrast, biomarkers that directly reflect the level of neuronal activity and real-time drug-induced changes in this activity offer superior tools for therapeutic evaluation. There are two practical options presently available to assess neuronal activity and monitor electrical activity and/or metabolic flux. Foremost are EEG-based measurements including basal state analysis and ERPs. Spectral analyses of resting EEGs from dementia patients generally exhibit increased power in lower frequency waves and a decline in higher frequencies ${ }^{[48]}$. Putative procognitive molecules shift the pattern of both low and high frequency waveforms toward normal patterns indicating improvements in synaptic function. Second, and perhaps more useful as drug evaluation tools, are ERPs. ERPs are time-locked potentials initiated by sensory, motor, or cognitive stimuli that represent the summed value of synchronized excitatory and inhibitory postsynaptic potentials, and as such are a measure of overall synaptic activity. The latencies of these potentials increase significantly in dementia patients suggesting degraded synaptic communication. Of the various ERPs studied, P300's ability to reflect the encoding of attention and working memory processes appears to be the most clinically relevant. Specifically, decreases in P300 amplitude and longer latencies indicate a decline in cognition ${ }^{[49]}$.

An alternative to EEG-based electrical markers that provide a real-time signature of neuronal activity are metabolic biomarkers that reflect the energy needs of neurons, which are in turn dependent upon their activity. Most common is the use of 2-deoxy-2-(18F) fluoro-D-glucose positron emission tomography (PET), which measures energy demand by trapping a non-metabolizable glucose analog that carries a PET label. Perhaps less direct, and another step removed from real-time brain activity, are blood-flow measurements. Blood flow is dependent on oxygen and nutrient demand which is driven by energy utilization and neuronal activity. Alzheimer's patients typically exhibit significantly decreased cerebral blood flow ${ }^{[50]}$. The complication in using blood-flow measurements as an evaluation tool concerns the potential role of vascular insufficiency as a contributor to some forms of dementia.

Major classes of drugs currently in clinical trials include neuroprotectants, stem cell therapies, tau vaccines, humanized antibodies, metabolic modulators, neurotransmitter modulators, DNA modifications, antihypertensives, NSAIDs, and a host of various "remedy" and vitamin treatments ${ }^{[2]}$. A first step in evaluating these new approaches and their probability of success may be to align the time course of useful ADassociated biomarkers with the posited treatment strategy. As discussed above, EEG-based measurements, including basal state analyses and ERPs, may be useful as tools to evaluate the therapeutic efficacy of these compounds.

A treatment strategy designed to significantly delay the symptoms and pathology of this disease could maintain the patient's quality of life and reduce health care costs. It has been estimated that an onset 
delay of 5 years would reduce the number of diagnosed patients by upwards of $50 \%^{[1,52]}$. Research efforts must continue with the ultimate goal of preventing neuronal damage and preserving normal cognitive functioning. However, efforts must also focus on the interim strategy to slow neuron losses in brain structures impacted including neocortices, hippocampus, locus ceruleus and the nucleus basalis of Meynert by stimulating synaptogenesis, neurogenesis and reducing neuroinflammation and oxidative stress. Such a strategy could significantly offset major memory-related symptoms. There are several neurotrophic agents capable of initiating such beneficial processes including NGF, BDNF, neurotrophin-3 and neurotrophin- $4 / 5^{[53-55]}$. There is an additional overlooked neurotrophin, HGF, which acts via the Met receptor. The HGF/Met system has been offered as a promising target for the treatment of $\mathrm{AD}^{[21,31,56,57]}$. The next section discusses criteria regarding the design and development of drugs to treat $\mathrm{AD}$.

\section{DRUG DESIGN CHALLENGES}

Several issues must be addressed concerning the development of an efficacious drug to treat AD: (1) the drug must penetrate the BBB to influence damaged neurons in memory-associated brain structures. This is may be a problem with some drugs that pass with difficulty or require piggy-back, nano- or viral technologies; (2) the half-life of the compound must be of sufficient duration to maintain a therapeutic level; (3) the avenue of drug delivery must be convenient for use by the patient and/or caregiver. This suggests oral, cutaneous (patch) or subcutaneous (as with pen delivery) routes. Once the drug satisfies these challenges it is preferable that it possess the following important characteristics; (4) the ability to promote stem cell proliferation, differentiation, and neurogenesis, and encourage synaptogenesis in memory related brain structures; (5) evidence neuroprotection especially against neuroinflammation, oxidative stressinduced damage and decreased cerebral blood flow; (6) facilitate LTP, memory consolidation and retrieval; and (7) delay, and hopefully prevent, the onset of MCI and cognitive dysfunctions associated with AD.

There are few neurotransmitters, neuromodulators or growth factors capable of satisfying the above listed criteria and preventing $\mathrm{AD}$-associated dysfunctions. However, as earlier suggested neurotrophic agents possess characteristics that make them excellent candidates. Of these BDNF has received considerable attention regarding depression and stress ${ }^{[58]}$ and $\mathrm{AD}^{[55,59,60]}$. However, neurotrophins have had little success in clinical trials directed at neurodegenerative diseases due to their poor pharmokinetic profile and large molecular weight that significantly impedes penetration of the $\mathrm{BBB}^{[61,62]}$. Jang et al.$^{[61,63]}$ have reported that the small molecule antidepressant drug amitriptyline is capable of binding to the BDNF target Trk receptor, induce receptor dimerization, and autophosphorylation. Receptor dimerization is a prerequisite to activation of neurotrophins and downstream signaling. Thus, the use of amitriptyline may serve as a "shortcut" past BDNF to receptor activation and have a positive impact against $\mathrm{AD}$. This drug is being tested in clinical trials conducted with depressed and chronic pain patients but not $\mathrm{AD}^{[29]}$. The neurotrophin, HGF, acts via the Met receptor. When activated the HGF/Met system has been shown to stimulate mitogenesis, motogenesis, morphogenesis and protect against tissue insults in several cellular targets including epithelial, endothelial, and neurons. This system has been offered as a target for the treatment of AD and is discussed in the next section ${ }^{[21,31,50,51]}$.

\section{DEVELOPMENT OF SMALL MOLECULES DESIGNED TO ACTIVATE THE HGF/MET SYSTEM}

In the 1990s members of our laboratory began testing the effectiveness of angiotensin IV (AngIV) and metabolically stable AngIV analogs to facilitate memory in animal models of AD [Figure 1]. In an effort to develop a useful drug we synthesized a number of AngIV-based compounds possessing extended halflives ${ }^{[64,65]}$. Our work resulted in one receptor agonist, $\mathrm{Nle}^{1}$-AngIV and two receptor antagonists, divalinalAngIV and norleual-AngIV ${ }^{[6-69]}$. We determined that Nle ${ }^{1}$-AngIV improved memory in animal models of $\mathrm{AD}$. This nootropic effect derived from its $\mathrm{N}$-terminal region given that tetra- and tripeptide fragments of $\mathrm{Nle}^{1}$-AngIV were also capable of overcoming scopolamine-induced amnesia ${ }^{[70-72]}$. In addition, $\mathrm{Nle}^{1}$-AngIV 


\section{Angiotensinogen}

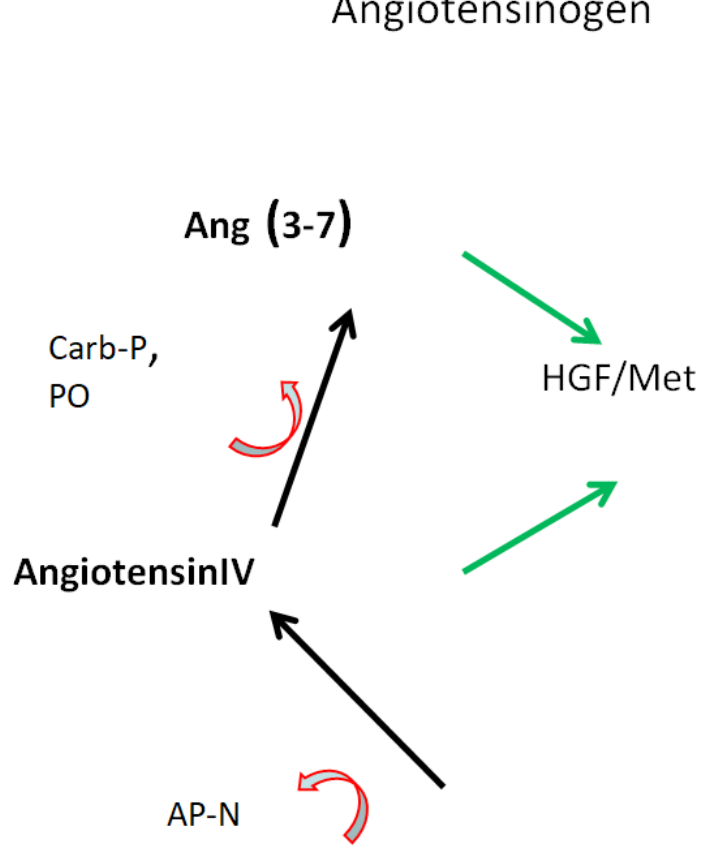

AngiotensinlII

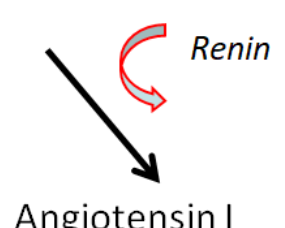

Angiotensin I
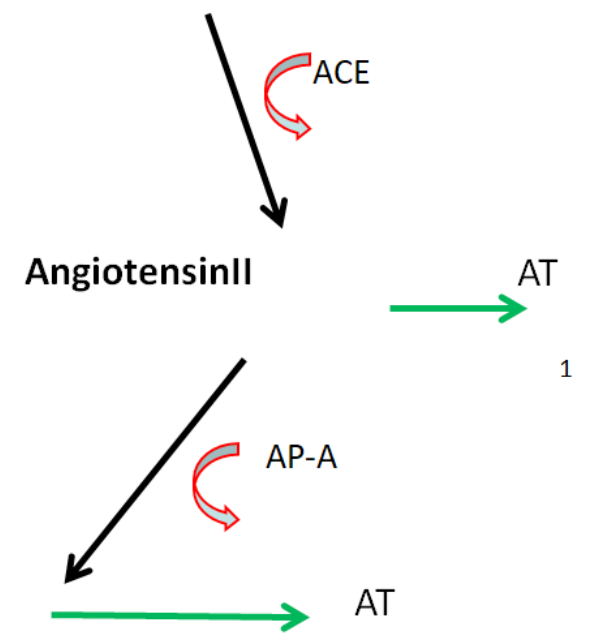

2

Figure 1. The biologically active ligands (bold), enzymes, and receptor proteins of the angiotensinogen-renin-angiotensin pathway that regulate angiotensin-based physiologies and behaviors. Angiotensinogen is a precursor protein consisting of 255 amino acids. Angiotensin II (Asp-Arg-Val-Tyr-lle-His-Pro-Phe) binds at the AT receptor subtype and mediates systemic blood pressure, body water/ electrolyte balance and influences cerebral blood flow, cerebroprotection, seizure, stress and depression. Angiotensin III (Arg-Val-Tyrlle-His-Pro-Phe) binds at the $\mathrm{AT}_{1}$ but primarily at the $\mathrm{AT}_{2}$ receptor subtype and modulates cell proliferation, cell differentiation, and apoptosis. This system also encourages some cellular regenerative processes and opposes most actions initiated by the angiotensin II/ $\mathrm{AT}_{1}$ receptor system. Angiotensin IV (Val-Tyr-lle-His-Pro-Phe) and Angiotensin (3-7: Val-Tyr-lle-His-Pro) bind at HGF thus stimulating dimerization a prerequisite to autophosphorylation of the Met receptor. This system mediates neuroprotection, synaptogenesis, cerebral blood flow, long-term potentiation and memory consolidation and retrieval. Both that $\mathrm{AT}_{1}$ and $\mathrm{AT}_{2}$ receptor subtypes are 7-transmembrane domain G-protein coupled receptors with molecular weights of $40-42 \mathrm{kDa}$. The Met receptor is a dimer as seen in growth factors, with a molecular weight of 160-190 kDa. ACE: angiotensin converting enzyme; AP-A: aminopeptidase A; AP-N: aminopeptidase N; Carb-P: carboxypeptidases P; HGF: hepatocyte growth factor; PO: propyl oligopeptidase

and these shorter fragments, facilitated hippocampal synaptic connectivity via synaptogenesis ${ }^{[70]}$. Synaptic functionality of these synapses was demonstrated via the presence of analog-induced spinogenesis, and the colocalization of synaptic markers in newly formed dendritic spines which were coupled with enhanced miniature excitatory postsynaptic currents. With these results in hand we became convinced that a clinically useful small molecule analog could be designed that possessed the important characteristics of metabolic stability, extended half-live, BBB penetrability and the capability to enhance cognitive functioning. Continued efforts resulted in an analog characterized by increased hydrophobicity, decreased hydrogen bonding potential coupled with increased metabolic stability bonding potential, and significantly increased metabolic stability ${ }^{[6,72]}$. This small molecule, dihexa, penetrates the BBB, induces spinogenesis/ synaptogenesis at picomolar concentrations ${ }^{[70]}$, and is slowly cleared from the blood (plasma stability $t_{1 / 2}=$ $335.5 \mathrm{~min})^{[73]}$. Also, dihexa can be delivered via parenteral routes of administration.

Dihexa displays several important characteristics. It binds with high affinity to HGF inducing dimerization and Met phosphorylation in the presence of subthreshold levels of HGF [Figure 2] ${ }^{[74]}$. High affinity binding sites are located at two sites on the $\alpha$-chain, specifically at the $\mathrm{N}$-terminal domain and the first Kringle domain. Binding sites with lower affinities are also located on the second and fourth Kringle domains but 


\section{A. HGF}

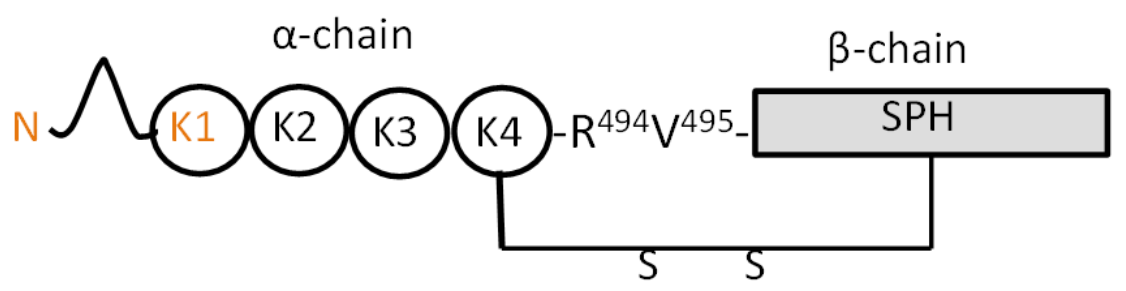

B. Met receptor

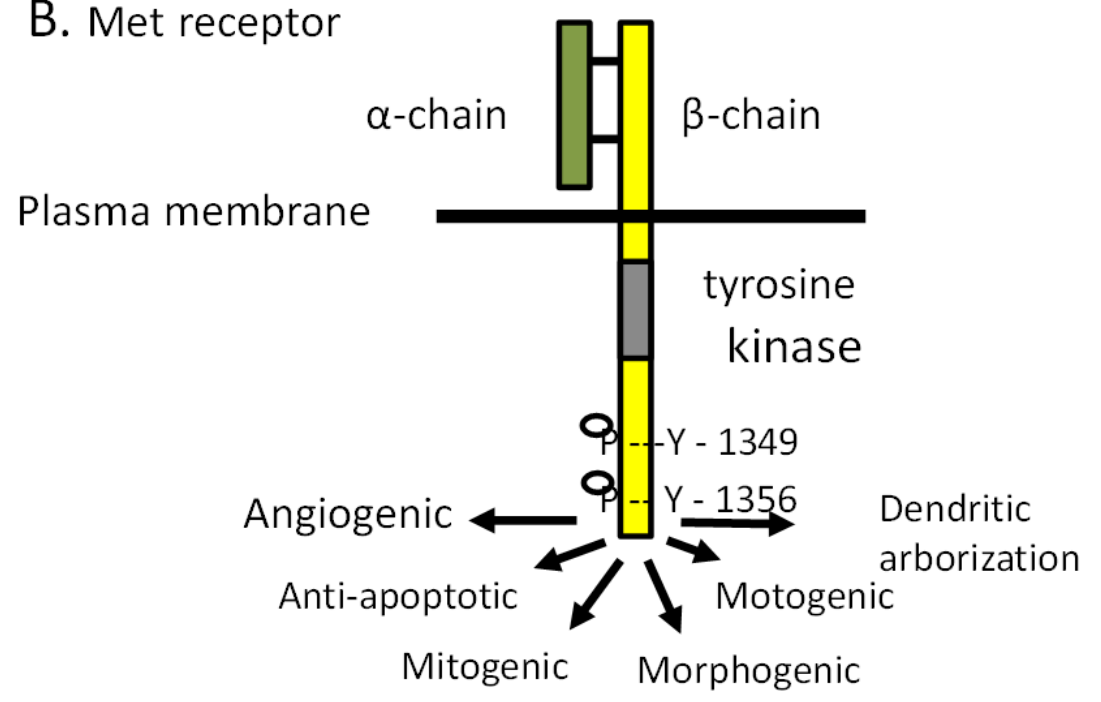

Figure 2. (A) Structure of HGF consisting of a $\alpha$-chain ( $69 \mathrm{kDa}$ ) including four Kringle domains and a $\beta$-chain ( $43 \mathrm{kDa}$ ) including a serine proteinase homology (SPH) domain, linked by disulfide bonds (S). High affinity binding sites are located at the $\mathrm{N}$-terminal domain and the first Kringle domain of the $\alpha$-chain; (B) Structure and basic functions of the Met receptor consisting of a $\alpha$-chain ( $50 \mathrm{kDa}$ ) and a $\beta$-chain $(140 \mathrm{kDa})$ linked by disulfide bonds. HGF binds to the Met receptor resulting in tyrosine phosphorylation leading to the activation of a number of biological activities including those listed plus anti-neuroinflammation and oxidative stress, increased cerebral blood flow and synaptogenesis, and facilitated long-term potentiation and memory. HGF: hepatocyte growth factor

appear to be of less importance ${ }^{[75-77]}$. Dihexa also stimulates hippocampal spinogenesis and synaptogenesis equivalent with HGF. Treatment with an HGF antagonist, "hinge", as well as a short hairpin RNA directed at Met, significantly inhibited these actions. Further, dihexa facilitated memory consolidation and retrieval in the scopolamine-induced amnesic rat model of $\mathrm{AD}$, as well as in memory compromised aged rats as measured using the Morris water maze task of spatial memory ${ }^{[73]}$. Taken together these results indicate that dihexa can reverse the memory deficits induced by scopolamine and aging.

The brain HGF/Met system appears to be designed to respond to injury as seen in stroke and neurodegenerative diseases by facilitating synaptic plasticity and neurogenesis. This hypothesis is supported by elevations in brain HGF levels measured in several degenerative diseases including amyotrophic lateral sclerosis, multiple sclerosis, Parkinson's disease, spinal cord injury and $\mathrm{AD}^{[78-82]}$. Although brain HGF levels are initially elevated in $\mathrm{AD}$ patients, they do not appear to be sustained.

\section{CONCLUSION}

Progress is being made concerning early detection of MCI. The development of a new efficacious drug to delay, and hopefully prevent, the onset of $\mathrm{AD}$ symptoms must catch up with these efforts. This will require a shift in our thinking. This shift is supported by the following facts and conclusions from past findings: (1) $\beta$-amyloid-induced plaques and neurofibrillary tangles define $\mathrm{AD}$ but may not cause it. These 
cellular markers are likely consequential to other deleterious dysfunctions; (2) efforts to develop drugs to rid neurons of amyloid plaque buildup have thus far failed to improve cognitive processing. It is likely that current efforts to prevent neurofibrillary tangles will also fall short regarding improved memory functioning; (3) Alzheimer's disease has a multitude of potential causes. These include, but are not limited to, genetic predisposition, neuroinflammation, head trauma, untreated hypertension, diabetes, Parkinson's disease, HIV infection and normal aging. It may be necessary to attack each of these likely causes with separate drug development programs; (4) presently available drugs do not promote synaptogenesis of existing neurons. Loss of synaptic connections is a major cause of neuronal apoptosis and discourages neurogenesis; (5) current research must focus on the development of drugs that penetrate the BBB, facilitate cognitive processing, and protect against the loss of synapses and neurons; (6) neurotrophic agents offer the ability to facilitate synaptogenesis, neurogenesis and neuroprotection thus greater research attention must be directed toward creating small molecule analogs designed to penetrate the $\mathrm{BBB}$ and activate these brain systems; and (7) it is likely that a successful approach to treating $\mathrm{AD}$ will require several different "multiple target directed ligands" (MTDLs). Neurotrophic small molecule analogs may be useful in configuring such strategies.

\section{DECLARATIONS}

\section{Authors' contributions}

Designed the paper, selected and prepared the references, prepared the figures, wrote the manuscript: Wright JW

Prepared the references, wrote the manuscript: Harding JW

\section{Availability of data and materials}

Not applicable.

\section{Financial support and sponsorship}

None.

\section{Conflicts of interest}

Both authors declare that there is no conflict of interest.

\section{Ethical approval and consent to participate}

All experiments utilizing animal models conducted in our laboratory and referred to in this review adhered to the Guidelines for the Care and Use of Laboratory Animals as required by the National Institutes of Health (NIH Publication No. 80-23), and the protocols were approved by the Washington State University Institutional Animal Care and Use Committee.

\section{Consent for publication}

Not applicable.

\section{Copyright}

(c) The Author(s) 2021.

\section{REFERENCES}

1. Berrios GE. Alzheimer's disease: a conceptual history. Inter J Geriatric 1990;5:355-65.

2. Hardy J, Selkoe DJ. The amyloid hypothesis of Alzheimer's disease: progress and problems on the road to therapeutics. Science 2002;297:353-6.

3. McGeer EG, McGeer PL. Inflammatory processes in Alzheimer's disease. Prog Neuropsychopharmacol Biol Psychiaty 2003;27:741-9.

4. Gorelick PB, Scuteri A, Black SE, Decaarli C, Greenberg SM, et al. Vascular contributions to cognitive impairment and dementia: a statement for healthcare professionals from the American heart association/American stroke association. Stroke 2011;42:2672-713. 
5. Serrano-Pozo A, Frosch MP, Masliah E, Hyman BT. Neuropathological alterations in Alzheimer disease. Cold Spring Harb Perspect Med 2011;1:a006189.

6. Alzheimer A. Über eine eigenartige Erkrankung der Himrinde. Allgemeine Zeitschrift fur Psychiatrie ünd phychish-Gerichtliche Medizin (Berlin) 1907;64:146-8.

7. Stelzmann RA, Schnitzlein HN, Murtagh FR. An english translation of Alzhimer's 1907 paper "Uber eine eigenantrige Erkenkung der Hirnrinde. Clin Anat 1995;8:429-31.

8. Mufson EJ, Counts SE, Ginsberg ST, Mahady L, Perez SE, et al. Nerve growth factor pathobiology during the progression of Alzheimer's disease. Front Neurosci 2019;13:533.

9. Hampel H, Mesulam, MM, Cuello AC, Farlow MR, Giacobini E, et al. The cholinergic system in the pathophysiology and treatment of Alzheimer's disease. Brain 2018;141:1917-33.

10. Alzheimer's Association, Available from: www.alz.org/. [Last accessed on 24 Jul 2020]

11. Irwin K, Sexton C, Daniel T, Lawlor B, Naci L. Healthy aging and dementia: two roads diverging in midlife? Front Aging Neurosci 2018;20:275-95.

12. Wimo A, Guerchet M, Ali GC, Wu YT, Prina AM, et al. The worldwide costs of dementia 2015 and comparisons with 2010. Alzheimers Dement 2017;13:1-7.

13. Prince M, Bryce R, Albanese E, Wimo A, Rieiro W, et al. The global prevalence of dementia: A systematic review and meta-analysis. Alzheimers Dement 2013;9:63-75.

14. Geral C, Angelova A, Lesieur S. From molecular to nanotechnology strategies for delivery of neurotrophins: Emphasis on brain-derived neurotrophic factor (BDNF). Pharmaceutics 2013;5:127-67.

15. Mitra S, Behbahani H, Eriksdotter M. Innovative therapy for Alzheimer's disease-With focus on biodelivery of NGF. Front Neurosci 2019;13:38.

16. Rafii MS, Tuszynski MH, Thomas RG, Barba D, Brewer JB, et al. Adeno-associated viral vector (Serotype 2)-nerve growth factor for patients with Alzheimer disease. JAMA Neurol 2018;5:834-41.

17. Palasz E, Wysocka A, Gasiorowska A, Chalimoniuk M, Niewiadomski W, et al. BDNF as a promising therapeutic agent in Parkinson's disease. Int J Mol Sci 2020;21:1170.

18. Massa SM, Yang T, Xie Y, Shi J, Bilgen M, et al. Small molecule BDNF mimetics activate TrkB signaling and prevent neuronal degeneration in rodents. J Clin Invest 2010;120:1774-85.

19. Albiston AL, Diwakaria S, Fernando RN, Mountford SJ, Yeatman H, et al. Identification and development of specific inhibitors for insulin-regulated aminopeptidase as a new class of cognitive enhancers. Br J Pharmacol 2011;164:37-47.

20. Briggs R, Kennelly SP, O’Neill DO. Drug treatments in Alzheimer's disease. Clin Med 2016;16:247-53.

21. Wright JW, Kawas LH, Harding JW. A role for the brain RAS in Alzheimer's and Parkinson's diseases. Front Endocrin 2013;4:158.

22. Melnikova I. Therapies for Alzheimer's disease. Nat Rev Drug Discov 2007;6:341-2.

23. Thomas SJ, Grossberg GT. Memantine: a review of studies into its safety and efficacy in treating Alzheimer's disease and other dementias. Clin Interv Aging 2009;4:367-77.

24. Vasser R. BACE1 inhibitor drugs in clinical trials for Alzheimer's disease. Alzheimers Res Ther 2014;6:89-103.

25. Prins ND, Scheltens P. Treating Alzheimer's disease with monoclonal antibodies: current status and outlook for the future. Alzheimers Res Ther 2013;5:56-66.

26. Salloway S, Sperling R, Fox NC, Blennow K, Klunk W, et al. Two phase 3 trials of bapineuzumab in mild-to-moderate Alzheimer's disease. N Engl J Med 2014;370:322-33.

27. Stansberry Investment Advisor. London: Alzheimer's disease Conference, July 19, 2017.

28. Wischik CM, Staff RT, Wischik DJ, Bentham P, Murray AD, et al. Tau aggregation inhibitor therapy: An exploratory phase 2 study in mild to moderate Alzheimer's disease. J Alzheimers Dis 2015;44:705-20.

29. U.S. National Library of Medicine. ClinicalTrials.gov website. Available from: //clinicaltrials.gov/. [Last accessed on 24 Jul 2017]

30. Cummings JL, Morstorf T, Zhong K. Alzheimer's disease drug-development pipeline: few candidates, frequent failures. Alzheimers Res Ther 2014;6:37-45.

31. Wright JW, Harding JW. Contributions by the brain renin-angiotensin system to memory, cognition, and Alzheimer's disease. J Alzheimers Dis 2019;67:469-80.

32. Braak H, Braak E. Frequency of stages of Alzheimer-related lesions in different age categories. Neurobiol Aging 1997;18:351-7.

33. De la Torre JC. Alzheimer's disease: How does it start? J Alzheimers Dis 2002;4:497-512.

34. Douchamps V, Mathis C. A second wind for the cholinergic system in Alzheimer's therapy. Behav Pharmacol 2017;28:112-23.

35. Albayram O, Herbert MK, Kondo A, Tsai CY, Baxley S, et al. Function and regulation of tau conformations in the development and treatment of traumatic brain injury and neurodegeneration. Cell Biosci 2016;6:59.

36. Mez J, Daneshyar DH, Kiernan PT, Abdolmohammadi B, Alarez VE, et al. Clinicopathological evaluation of chronic traumatic encephalopathy in players of American football. JAMA 2017;318:360-70.

37. Randolph C, Karantzoulis S, Guskiewicz K. Prevalence and characterization of mild cognitive impairment in retired national football league players. J Int Neuropsychol Soc 2013;19:873-80.

38. FDA-NIH biomarker working group, BEST (Biomarkers, EndpointS, and other Tools) resource, Silver Spring (MD): Food and Drug Administration; ssary, 2016. Available from: https://www.ncbi.nlm.nih.gov/books/NBK338448. [Last accessed on 24 Jul 2017]

39. Calsolaro V, Edison P. Neuroinflammation in Alzheimer's disease: current evidence and future directions. Alzheimers Dement 2016;12:719-32. 
40. Labzin LI, Heneka MT, Latz E. Innate immunity and neurodegeneration. Annu Rev Med 2018;69:437-49.

41. Mawanda F, Wallace R. Can infections cause Alzheimer's disease? Epidemiol Rev 2013;35:161-80.

42. Tramutola A, Lanzillotta C, Perluigi M, Allan Butterfield D. Oxidative stress, protein modification and Alzheimer disease. Bran Res Bull 2017;133:88-96.

43. Abbott A. The brain inflamed. Nature 2018;556:426-8.

44. Holtman IR, Raj DD, Miller JA, Schaafsma W, Yin Z, et al. Induction of a common microglia gene expression signature by aging and neurodegenerative conditions: a co-expression meta-analysis. Acta Neuropathol Commun 2015;3:31.

45. Krasemann S, Madore C, Cialic R, Raufeld C, Calcagno N, et al. The TREM2-APOE pathway drives the transcriptional phenotype of dysfunctional microglia in neurodegenerative diseases. Immunity 2017;47:566-81.

46. Ardura-Fabregat A, Boddeke EW, Boza-Serrano A, Brioschi S, Castro-Gomez S, et al. Targeting neuroinflammation to treat Alzheimer's disease. CNS Drugs 2017;31:1057-82.

47. Fortea J, Vilaplana E, Carmona-Iragui M, Benejam B, Videla L, et al. Clinical and biomarker changes of Alzheimer's disease in adults with Down syndrome: a cross-sectional study. Lancet 2020;395:1988-97.

48. Nimmrich V, Draguhn A, Axmacher N. Neuronal network oscillations in neurodegenerative diseases. Neuromol Med 2015;17:270-84.

49. Ally BA, McKeever JD, Waring JD, Budson AE. Preserved frontal memorial processing for pictures in patients with mild cognitive impairment. Neuropsychologia 2009;47:2044-55.

50. Benedictus MR, Leeuwis AE, Binnewijzend MA, Kuijer JP, Scheltens P, et al. Lower cerebral blood flow is associated with faster cognitive decline in Alzheimer's disease. Eur Radiol 2017;27:1169-75.

51. De la Torre JC. Carotid artery ultrasound and echocardiography testing to lower the prevalence of Alzheimer's disease. J Stroke Cerebrovasc Dis 2009;18:319-28.

52. De la Torre JC. Are major dementias triggered by poor blood flow to the brain? Theoretical considerations. J Alzheimers Dis 2017;57:353-71.

53. Boyce VS, Mendell LM. Neurotrophins and spinal circuit function. Front Neural Circuits 2014;8:59-67.

54. Lu B, Nagappan G, Lu Y. BDNF and synaptic plasticity, cognitive function, and dysfunction. Handb Exp Pharmacol 2014;220:220-3.

55. West AF, Pruunslid P, Timmusk T. Neurotrophins: transcription and translation. Handb Exp Pharmacol 2014;220:67-100.

56. Hamasaki H, Honda H, Suzuki SO, Hokama M, Kiyohara Y, et al. Down-regulation of MET in hippocampal neurons of Alzheimer's disease brains. Neuropathology 2014;34:284-90.

57. Sharma SK. Hepatocyte growth factor in synaptic plasticity and Alzheimer's disease. Sci World J 2010;10:457-61.

58. Gibon J, Barker PA. Neurotrophins and proneuotrophins: Focus on synaptic activity and plasticity in the brain. Neuroscientist 2017;23:587-604.

59. Chen G, Shu H, Chen G, Ward BD, Antuono PG, et al. Staging Alzheimer's disease risk by sequencing brain function and structure, cerebrospinal fluid, and cognition biomarkers. J Alzheimers Dis 2016;54:983-93.

60. Wang R, Holsinger RMD. Exercise-induced brain-derived neurotrophic factor expression: Therapeutic implications for Alzheimer's dementia. Aging Res Rev 2018;48:109-21.

61. Jang SW, Liu X, Yepes M, Shephard KR, Miller GW, et al. A selective TrkB agonist with potent neurotrophic activities by 7,8-dihydroxyflavone. Proc Natl Acad Sci USA 2010;107:2687-92.

62. O'Leary PD, Hughes RA. Design of potent peptide mimetics of brain-derived neurotrophic factor. J Biol Chem 2003;278:25738-44.

63. Jang SW, Liu X, Chan CB, Weinshenker D, Hall RA, et al. Amitriptyline is a TrkA and TrkB receptor agonist that promotes TrkA/TrkB heterodimerization and has potent neurotrophic activity. Chem Biol 2009;16:644-65

64. Sardinia, MF, Hanesworth JM, Krebs LT, Harding JW. AT4 receptor binding characteristics: D-amino acid- and glycine-substituted peptides. Peptides 1993;14:949-54.

65. Sardinia MF, Hanesworth JM, Krishnan F, Harding JW. AT4 receptor structure-binding relationship: N-terminal-modified angiotensin IV analogues. Peptides 1994;15:1399-406.

66. Kawas LH, McCoy AT, Yamamoto BJ, Wright JW, Harding JW. Development of angiotensin IV analogs as hepatocyte growth factor/Met modifiers. J Pharmacol Exp Ther 2012;340:539-48.

67. Kramár EA, Armtrong DL, Ikeda S, Wayner MJ, Harding JW, et al. The effects of angiotensin IV analogs on long-term potentiation within the CA1 region of the hippocampus in vitro. Brain Res 2001;897:114-21.

68. Krebs LT, Kramar EA, Hanesworth JM, Sardinia MF, Ball AE, et al. Characterization of the binding properties and physiological action of divalinal-angiotensin IV, a putative AT4 receptor antagonist. Regul Pept 1996;67:123-30.

69. Yamamoto BJ, Elias PD, Masino JA, Hudson BD, McCoy AT, et al. The angiotensin IV analog Nle-Tyr-Leu- $\psi-\left(\mathrm{CH}_{2}-\mathrm{NH}_{2}\right)^{3-4}-\mathrm{His}-\mathrm{Pro}-\mathrm{Phe}$ (Norleual) can act as a hepatocyte growth factor/c-Met inhibitor. J Pharmacol Exp Ther 2010;33:161-73.

70. Benoist CC, Wright JW, Zhu M, Appleyard SM, Wayman GA, et al. Facilitation of hippocampal synaptogenesis and spatial memory by C-terminal truncated Nle1-angiotensin IV analogs. J Pharmacol Exp Ther 2011;339:35-44.

71. Wright JW, Harding JW. The brain angiotensin IV/AT ${ }_{4}$ receptor system as a new target for the treatment of Alzheimer's disease. Drug Dev Res 2009;70:472-80.

72. Wright JW, Harding JW. The brain hepatocyte growth factor/c-Met receptor system: a new target for the treatment of Alzheimer's disease. J Alzheimers Dis 2015;45:985-1000.

73. McCoy AT, Benoist CC, Wright JW, Kawas LH, Bule-Ghogare JM, et al. Evaluation of metabolically stabilized angiotensin IV analogs as procognitive/antidementia agents. J Pharmacol Exp Ther 2013;344:141-54.

74. Benoist CC, Kawas LH, Zhu M, Tyson KA, Stillmaker L, et al. The pro-cognitive and synaptogenic effects of angiotensin IV-derived 
peptides are dependent on activation of the hepatocyte growth factor/c-Met system. J Pharmacol Exp Ther 2014;351:390-402.

75. Holmes O, Pillozzi S, Deakin JA, Carafoli F, Kemp L, et al. Insights into the structure/function of hepatocyte growth factor/scatter factor from studies with individual domains. J Mol Biol 2007;367:395-408.

76. Nakamura T, Mizuno S. The discovery of hepatocyte growth factor (HGF) and its significance for cell biology, life sciences and clinical medicine. Proc Jpn Acad Ser B Phys Biol Sci 2010;86:588-610.

77. Wright JW, Kawas LH, Harding JW. The development of small molecule angiotensin IV analogs to treat Alzheimer's and Parkinson's diseases. Prog Neurobiol 2015;125:26-46.

78. Choi W, Lee J, Lee J, Lee SH, Dim S. Hepatocyte growth factor regulates macrophage transition to the M2 phenotype and promotes murine skeletal muscle regeneration. Front Physiol 2019;10:914.

79. Kato S, Funakoshi H, Nakamura T, Kato M, Nakano I, et al. Expression of hepatocyte growth factor and c-Met in the anterior horn cells of the spinal cord in the patients with amyotrophic lateral sclerosis (ALS): immunohistochemical studies on sporadic ALS and familial ALS with superoxide dismutase 1 gene mutation. Acta Neurophathol 2003;106:112-20.

80. Muller AM, Jun E, Conlon H, Sadiq SA. Cerebrospinal hepatocyte growth factor levels correlate negatively with disease activity in multiple sclerosis. J Neuroimmunol 2012;251:80-6.

81. Salehi Z, Rajael F. Expression of hepatocyte growth factor in the serum and cerebrospinal fluid of patients with Parkinson's disease. J Clin Neurosci 2010;17:1553-6.

82. Shimamura M, Sata N, Sataq M, Wakayama K, Ogihara T, et al. Expression of hepatocyte growth factor and c-Met after spinal cord injury in rats. Brain Res 2007;1151:188-94. 